\title{
The Stepanov Differentiability Theorem in Metric Measure Spaces
}

\author{
By Zoltán M. Balogh, Kevin Rogovin, and Thomas Zürcher
}

\begin{abstract}
We extend Cheeger's theorem on differentiability of Lipschitz functions in metric measure spaces to the class of functions satisfying Stepanov's condition. As a consequence, we obtain the analogue of Calderon's differentiability theorem of Sobolev functions in metric measure spaces satisfying a Poincaré inequality.
\end{abstract}

\section{Introduction}

The classical result of Rademacher (see e.g., [7], 3.1.2) states that a Lipschitz function $f: \mathbb{R}^{n} \rightarrow \mathbb{R}$ is differentiable almost everywhere (in the sense of the Lebesgue measure). There are at least two important generalizations of Rademacher's theorem. The first one is due to Stepanov (see e.g., [8], 3.1.8) and states that $f: \mathbb{R}^{n} \rightarrow \mathbb{R}$ is differentiable at a.e. $x \in S(f)$, where

$$
S(f):=\left\{x \in \mathbb{R}^{n}: \limsup _{y \rightarrow x} \frac{|f(y)-f(x)|}{|y-x|}<\infty\right\} .
$$

The second generalization is the theorem of Calderon (see e.g., [13], 6.17) which claims the a.e. differentiability of Sobolev functions $f \in W^{1, p}\left(\mathbb{R}^{n}\right)$ for $p>n$. These results are major comerstones of analysis in Euclidean spaces with applications in geometric measure theory [8] and differentiability of quasiconformal and quasiregular mappings [23].

Recent years have seen an intense ongoing research activity in extending classical results of analysis in Euclidean spaces to the setting of general metric-measure spaces. We do not intend to present a full list of achievements in this area, but we mention the works $[12,15,21,11,9]$, and [10] for results on Sobolev spaces; and works of [14] and [19] for results on quasiconformal mappings in this general framework. We refer to the monograph of Heinonen [13] for an overview of this development.

A major advance in this area of research was marked by the work of Cheeger [5] who extended Rademacher's differentiability theorem to the fairly large class of metric-measure spaces which

Math Subject Classifications. 28A15, 26B05.

Key Words and Phrases. Differentiability, Poincaré inequality, Sobolev function, metric space.

Acknowledgements and Notes. This research was supported by a grant from the Swiss NSF. 
satisfy a doubling condition and admit a Poincaré inequality. Cheeger's work has been recently extended to even more general spaces by Keith $[16,17]$.

The main result of the present article is a Cheeger-Stepanov type differentiability theorem in metric-measure spaces. Roughly speaking, we prove that in the presence of a doubling condition the Cheeger-Rademacher differentiability theorem implies a Stepanov-type differentiability result. As applications we obtain statements on differentiability of quasiconformal mappings and Sobolev functions in various general metric space settings.

This article is organized as follows. In the second section we recall some terminology and preliminary results. In Section 3 we state and prove the Cheeger-Stepanov differentiability theorem. In Section 4 we present results on differentiability of Sobolev functions and quasiconformal mappings as applications.

\section{Preliminaries}

Definition 2.1. Let $(X, d)$ be a metric space. For $x \in X$ and $r>0$ we let $\mathbf{B}(x, r)=\{y \in$ $X \mid d(x, y)<r)$ be the open ball of radius $r$ around $x$. We will write $\tau \mathbf{B}(x, r)$ to mean $\mathbf{B}(x, \tau r)$. A function $f:(X, d) \rightarrow(Y, \rho)$ between metric spaces is called $K$-Lipschitz if for each $x, y \in X$, $\rho(f(x) f(y)) \leq K d(x, y)$. We let $\operatorname{LIP}(f)$ be the infimum of such $K$. We let $\operatorname{LIP}(X)$ be the set of real valued Lipschitz function on $X$ where $\mathbb{R}$ is considered with the usual Euclidean metric.

Definition 2.2. A triple $(X, d, \mu)$ is called a metric measure space if $(X, d)$ is a metric space and $\mu$ is a Radon measure on $X$.

Following Cheeger [5], it is possible to define the notion of a differentiable structure in metric-measure spaces as follows.

Definition 2.3 (Strong measurable differentiable structure). $\quad$ Let $(X, d, \mu)$ be a metric measure space, let $\mathcal{C} \subset \operatorname{LIP}(X)$ be a vector space of functions, and let $\left\{\left(X_{\alpha}, \varphi_{\alpha}\right)\right\}$ be a countable collection such that each set $X_{\alpha} \subset X$ is measurable with positive measure, and such that each

$$
\varphi_{\alpha}=\left(\varphi_{\alpha}^{1}, \ldots, \varphi_{\alpha}^{N(\alpha)}\right): X \rightarrow \mathbb{R}^{N(\alpha)}
$$

is a function for some $N(\alpha) \in \mathbb{N} \cup\{0\}$, where $\varphi_{\alpha}^{i} \in \mathcal{C}$ for every $1 \leq i \leq N(\alpha)$. (Here $\varphi_{\alpha}$ will be viewed to be the empty function if $N(\alpha)=0$.) Then $\left\{\left(X_{\alpha}, \varphi_{\alpha}\right)\right\}$ is said to be a strong measurable differentiable structure for $(X, d, \mu)$ with respect to $\mathcal{C}$ if the following conditions are true.

(i) The sets $X_{\alpha}$ are pairwise disjoint and

$$
\mu\left(X \backslash \bigcup_{\alpha} X_{\alpha}\right)=0 .
$$

(ii) There exists a number $N \geq 0$ such that $N(\alpha) \leq N$ for every coordinate patch $\left(X_{\alpha}, \varphi_{\alpha}\right)$.

(iii) For every $f \in \mathcal{C}$ and coordinate patch $\left(X_{\alpha}, \varphi_{\alpha}\right)$, there exists a unique (up to a set of zero measure) measurable function $d^{\alpha} f: X_{\alpha} \rightarrow \mathbb{R}^{N(\alpha)}$ such that for $\mu$-almost every $x \in X_{\alpha}$,

$$
\lim _{\substack{y \rightarrow x \\ y \neq x}} \frac{\left|f(y)-f(x)-\left\langle d^{\alpha} f(x), \varphi_{\alpha}(y)-\varphi_{\alpha}(x)\right\rangle\right|}{d(y, x)}=0
$$

where $\langle\cdot, \cdot\rangle$ is the usual Euclidean inner-product on $R^{N(\alpha)}$. 
For those $x$ for which a $d^{\alpha} f(x)$ exists so that the relation (2.1) holds, we say that $f$ is differentiable at $x$.

Note that the existence of a strong measurable differentiable structure on a space $X$ with $\mathcal{C}=\operatorname{LIP}(X)$ contains in the definition itself a Rademacher-type differentiability in the sense of (2.1). If $X=\mathbb{R}^{n}, d$ is the standard Euclidean metric and $\mu$ is the Lebesgue measure on $X$, then the classical Rademacher's theorem implies that $X_{1}=\mathbb{R}^{n}$ and $\phi_{1}(x)=x$ defines a strong measurable differentiable structure for $\operatorname{LIP}\left(\mathbb{R}^{n}\right)$. The problem arises to find sufficient conditions to guarantee the existence of a strong measurable differentiable structure for $\operatorname{LIP}(X)$. The first result in this direction was given by Cheeger in his seminal article, [5]. To formulate Cheeger's result we introduce the following concepts.

Definition 2.4. A measure $\mu$ on a metric measure space $(X, d, \mu)$ is said to be doubling if $\mu$ is non trivial and there exists a constant $C>0$ such that

$$
\mu(2 \mathbf{B}) \leq C \mu(\mathbf{B})
$$

whenever $\mathbf{B}$ is a ball in $X$.

It turns out that for doubling measures the Lebesgue differentiation theorem holds, see [13], 1.8. More precisely, let us recall that for a given set $A \subseteq X$, we call $x \in A$ a point of density of $A$ if

$$
\lim _{r \rightarrow 0} \frac{\mu(A \cap \mathbf{B}(x, r))}{\mu(\mathbf{B}(x, r))}=1 .
$$

When $\mu$ is a doubling measure, $\mu$-a.e. $x \in A$ is a point of density of $A$. Moreover, for $f \in L^{p}(\mu)$, we call $x_{0}$ a Lebesgue point of $f$ if

$$
\limsup _{r \rightarrow 0} f_{\mathbf{B}\left(x_{0}, r\right)}\left|f(x)-f\left(x_{0}\right)\right|^{p} d \mu(x)=0,
$$

where for any set $S$, the average of $f$ over $S$ is defined as

$$
f_{S} f d \mu:=\frac{1}{\mu(S)} \int_{S} f d \mu
$$

When $\mu$ is doubling, if $f \in L^{p}(\mu)$ then $\mu$-a.e. $x$ is a Lebesgue point of $f$.

In addition to the doubling condition the following general version of the Poincaré inequality is a crucial property of a metric measure space.

Definition 2.5. Let $p \geq 1$. A metric measure space $(X, d, \mu)$ is said to satisfy a $(1, p)$-Poincaré inequality if there exist constants $C>0$ and $\tau \geq 1$ so that

$$
f_{\mathbf{B}}\left|u-u_{\mathbf{B}}\right| d \mu \leq C \operatorname{diam}(\mathbf{B})\left(f_{\tau \mathbf{B}} \rho^{p} d \mu\right)^{1 / p}
$$

whenever $u$ is a continuous function with upper gradient $\rho$ and $\mathbf{B}$ is a ball in $X$. We say $\rho$ is an upper gradient of $u$ if for each rectifiable curve $\gamma:[0, T] \rightarrow X$,

$$
|u(\gamma(T))-u(\gamma(0))| \leq \int_{\gamma} \rho d \mathcal{H}_{1}
$$

where $\mathcal{H}_{1}$ is the Hausdorff 1-measure in $X$. 
When a pair of function $(u, \rho)$ satisfies Equation (2.2) for all balls $\mathbf{B}$ of $X$, we say that the pair $(u, \rho)$ satisfies a $(1, p)$-Poincaré inequality.

The class of metric-measure spaces that satisfy the doubling condition and a Poincare inequality are the ones on which a considerable part of analysis from the Euclidean theory still holds [14, 15, 19, 21]. According to Cheeger's result [5], these conditions are also sufficient for the existence of a strong measurable differentiable structure.

Theorem 2.6. Let $(X, d, \mu)$ be a metric measure space with $\mu$ doubling that satisfies a $(1, p)$ Poincaré inequality for some $p \geq 1$. Then $(X, d, \mu)$ admits a strong measurable differentiable structure for $\operatorname{LIP}(X)$.

Moreover, according to Keith [16] the condition on the Poincaré inequality can be substantially relaxed. For his generalization, let us recall the definition of a chunky measure.

Definition 2.7. A measure $\mu$ on a metric space $(X, d)$ is called chunky if for $\mu$-a.e. $x \in X$, there exists a positive decreasing sequence $\left\{r_{n}\right\}$ converging to zero so that for each $\epsilon>0$ there exists $N \in \mathbb{N}$ for which

$$
\frac{\mu\left(\mathbf{B}\left(y, \epsilon r_{n}\right)\right)}{\mu\left(\mathbf{B}\left(x, r_{n}\right)\right)} \geq \frac{1}{N}
$$

whenever $n>N$ and $y \in \mathbf{B}\left(x, r_{n}\right)$.

Theorem 2.8. Let $(X, d, \mu)$ be a locally compact metric measure space with $X$ doubling and the measure $\mu$ is Radon and chunky. Let $K \geq 1$, and let $\mathcal{C} \subset \operatorname{LIP}(X)$ be a vector space of functions which satisfies the condition that for every $f \in \mathcal{C}$,

$$
\operatorname{Lip} f(x) \leq K \operatorname{lip} f(x),
$$

for almost every $x \in X$. Then $(X, d, \mu)$ admits a strong measurable differentiable structure with respect to $\mathcal{C}$. Moreover, if $(X, d, \mu)$ satisfies a Poincaré inequality, then there exists a constant $K>0$ so that each $f \in \operatorname{LIP}(X)$ satisfies (2.3) at $\mu$-a.e. $x \in X$.

Here, the upper- and lower-scaled oscillations of $f, \operatorname{Lip} f$ and lip $f$ are defined by Keith as

$$
\begin{gathered}
\operatorname{lip} f(x)=\liminf _{r \rightarrow 0} \sup _{y \in \mathbf{B}(x, r)} \frac{|f(x)-f(y)|}{r}, \\
\operatorname{Lip} f(x)=\limsup _{r \rightarrow 0} \sup _{y \in \mathbf{B}(x, r)} \frac{|f(x)-f(y)|}{r} .
\end{gathered}
$$

Note that $\operatorname{Lip} f\left(x_{0}\right)=\lim \sup _{x \rightarrow x_{0}} \frac{\left|f(x)-f\left(x_{0}\right)\right|}{d\left(x, x_{0}\right)}$ and thus Stepanov's theorem could be stated as $f: \mathbb{R}^{n} \rightarrow \mathbb{R}$ is differentiable for a.e. $x \in S(f)$, where

$$
S(f)=\left\{x \in \mathbb{R}^{n}: \operatorname{Lip} f(x)<\infty\right\} .
$$

It is an important observation to note that the above quantities do not change under restriction to positive measure sets. To be more precise, for a given subset $K$ of $X$, define the scaled oscillations of $f$ restricted to $K$ as

$$
\begin{aligned}
\operatorname{lip}_{K} f(x) & =\liminf \operatorname{in}_{r \rightarrow 0} \sup _{y \in K \cap \mathbf{B}(x, r)} \frac{|f(x)-f(y)|}{r}, \\
\operatorname{Lip}_{K} f(x) & =\lim \sup _{r \rightarrow 0} \sup _{y \in K \cap \mathbf{B}(x, r)} \frac{|f(x)-f(y)|}{r} .
\end{aligned}
$$


Proposition 2.9. Let $(X, d, \mu)$ be a metric-measure space where $\mu$ is a doubling measure. Let $K \subseteq X$ and $f \in \operatorname{LIP}(X)$. Then for each point of density $x_{0}$ of $K$,

$$
\begin{aligned}
\operatorname{Lip}_{K} f\left(x_{0}\right) & =\operatorname{Lip} f\left(x_{0}\right) \\
\operatorname{lip}_{K} f\left(x_{0}\right) & =\operatorname{lip} f\left(x_{0}\right) .
\end{aligned}
$$

In particular, for $\mu$-a.e. $x \in f^{-1}(0)$, Lip $f(x)=0$.

Proof. Let us start by the following direct consequence of the doubling condition for the measure $\mu$ (see e.g., [15], 14.6) according to which there exist constants $C>0$ and $Q>0$ depending only on the doubling constant for $\mu$ such that

$$
\frac{\mu(B(x, r))}{\mu\left(B\left(x_{0}, r_{0}\right)\right)} \geq \frac{1}{C}\left(\frac{r}{r_{0}}\right)^{Q}
$$

whenever $x \in B\left(x_{0}, r_{0}\right)$ and $r \leq r_{0}$.

The proof of the proposition is based on the following claim. If $x_{0} \in K \subset X$ is a point of density of $K$, then for each $\epsilon>0$ there exists $r>0$ so that for each $x \in \mathbf{B}\left(x_{0}, r\right)$, there exists $y \in K$ so that $d(y, x) \leq \epsilon d\left(x, x_{0}\right)$.

To prove the claim we assume by contradiction that there exists a sequence of points $\left(x_{n}\right)$ so that $x_{n} \rightarrow x_{0}$ and $\mathbf{B}\left(x_{n}, \varepsilon d\left(x_{n}, x_{0}\right)\right) \cap K=\emptyset$.

This implies

$$
\begin{aligned}
0 & =\lim _{r \rightarrow 0} \frac{\mu\left(\mathbf{B}\left(x_{0}, r\right) \cap K^{c}\right)}{\mu\left(\mathbf{B}\left(x_{0}, r\right)\right)} \\
& =\lim _{n \rightarrow \infty} \frac{\mu\left(\mathbf{B}\left(x_{0},(1+\varepsilon) d\left(x_{n}, x_{0}\right)\right) \cap K^{c}\right)}{\mu\left(\mathbf{B}\left(x_{0},(1+\varepsilon) d\left(x_{n}, x_{0}\right)\right)\right)} \\
& \geq \lim _{n \rightarrow \infty} \frac{\mu\left(\mathbf{B}\left(x_{n}, \varepsilon d\left(x_{n}, x_{0}\right)\right) \cap K^{c}\right)}{\mu\left(\mathbf{B}\left(x_{0},(1+\varepsilon) d\left(x_{n}, x_{0}\right)\right)\right)} \\
& =\lim _{n \rightarrow \infty} \frac{\mu\left(\mathbf{B}\left(x_{n}, \varepsilon d\left(x_{n}, x_{0}\right)\right)\right)}{\mu\left(\mathbf{B}\left(x_{0},(1+\varepsilon) d\left(x_{n}, x_{0}\right)\right)\right)}
\end{aligned}
$$

Since $\mu$ is a doubling measure we can use (2.4) to conclude that

$$
\begin{aligned}
0 & \geq \lim _{n \rightarrow \infty} \frac{1}{C}\left(\frac{\varepsilon d\left(x_{n}, x_{0}\right)}{(1+\varepsilon) d\left(x_{n}, x_{0}\right)}\right)^{Q} \\
& =\frac{1}{C}\left(\frac{\varepsilon}{1+\varepsilon}\right)^{Q}>0
\end{aligned}
$$

which is a contradiction.

Let $f \in \operatorname{LIP}(X)$, clearly $\operatorname{Lip}_{K} f(x) \leq \operatorname{Lip} f(x)$ and $\operatorname{lip}_{K} f(x) \leq \operatorname{lip} f(x)$ for all $x \in K$. Let $x_{0} \in K$ be a point of density of $K$ and $\epsilon>0$. For each $r>0$ let $x_{r} \in \mathbf{B}\left(x_{0}, r\right)$ so that

$$
\left|f\left(x_{r}\right)-f\left(x_{0}\right)\right| \geq-\epsilon r+\sup _{x \in \mathbf{B}\left(x_{0}, r\right)}\left|f(x)-f\left(x_{0}\right)\right| .
$$

Then from the above claim, for $r$ sufficiently small, there exists $y_{r} \in K$ so that $d\left(y_{r}, x_{r}\right) \leq$ 
$\epsilon d\left(x_{r}, x_{0}\right)$. Hence,

$$
\begin{aligned}
\operatorname{lip}_{K} f\left(x_{0}\right) & =\liminf _{r \rightarrow 0} \frac{1}{(1+\epsilon) r} \sup _{y \in K \cap \mathbf{B}\left(x_{0},(1+\epsilon) r\right)}\left|f(y)-f\left(x_{0}\right)\right| \\
& \geq \liminf _{r \rightarrow 0} \frac{\left|f\left(x_{0}\right)-f\left(y_{r}\right)\right|}{(1+\epsilon) r} \\
& \geq \liminf _{r \rightarrow 0} \frac{\left|f\left(x_{0}\right)-f\left(x_{r}\right)\right|-\left|f\left(x_{r}\right)-f\left(y_{r}\right)\right|}{(1+\epsilon) r} \\
& \geq \liminf _{r \rightarrow 0} \frac{1}{(1+\epsilon) r}\left(-\epsilon r+\sup _{y \in \mathbf{B}\left(x_{0}, r\right)}|f(x)-f(y)|-\epsilon r \operatorname{LIP}(f)\right) \\
& =\frac{1}{\epsilon+1} \operatorname{lip} f\left(x_{0}\right)-\frac{\epsilon}{1+\epsilon}(1+\operatorname{LIP}(f))
\end{aligned}
$$

which when we let $\epsilon \rightarrow 0$ implies that $\operatorname{lip}_{K} f\left(x_{0}\right) \geq \operatorname{lip} f\left(x_{0}\right)$. The same reasoning as above (by replacing $\lim$ inf with $\lim \sup$ ) gives us that $\operatorname{Lip}_{K} f\left(x_{0}\right) \geq \operatorname{Lip} f\left(x_{0}\right)$.

The second statement of the proposition is obtained from the first one by setting $K=$ $f^{-1}(0)$ and observing that $\operatorname{Lip}_{K} f(x)=0$ for all points $x \in K$.

Corollary 2.10. Let $(X, d, \mu)$ be a doubling metric measure space. Assume that there is a strong measurable differentiable structure $\left\{\left(X_{\alpha}, \varphi_{\alpha}\right)\right\}$ supported by $(X, d, \mu)$. Let $f: X \rightarrow \mathbb{R}$ be a Lipschitz function. Then at $\mu$-a.e. point $x$ where $f(x)=0$ we have $d^{\alpha} f(x)=0$.

Proof. Set $K:=f^{-1}(0)$. By Proposition 2.9 we know that at $\mu$-a.e. point of $K$ we have Lip $f(x)=0$. Take such a point and assume that $x \in X_{\alpha}$. It follows that

$$
\lim _{\substack{y \rightarrow x \\ y \neq x}} \frac{|f(y)-f(x)|}{d(y, x)} \leq \limsup _{\substack{y \rightarrow x \\ y \neq x}} \frac{|f(x)-f(y)|}{d(y, x)}=\operatorname{Lip} f(x)=0 .
$$

By Definition 2.3 the differential is unique up to a set of measure zero and therefore it follows that at $\mu$-a.e. point in $K$ the differential of $f$ is 0 .

Proposition 2.9 together with Keith's theorem, Theorem 2.8, imply the following.

Theorem 2.11. Let $(X, d, \mu)$ be a complete metric measure space with $\mu$ doubling on $X$ so that there exists a constant $C>0$ so that for each $f \in \operatorname{LIP}(X)$, and for $\mu$-a.e. $x$, Lip $f(x) \leq$ $C$ lip $f(x)$. Then for each closed subset $A$ of $X$ with $\mu(A)>0$, the metric measure space $(A, d, \mu)$ admits a strong measurable differentiable structure with respect to $\operatorname{LIP}(A)$.

Proof. Fix a measurable differentiable structure on $X$. For each $f \in \operatorname{LIP}(A)$, there exists $F \in \operatorname{LIP}(X)$ so that $\left.F\right|_{A}=f$. For points of density $x_{0}$ of $A$ we have by Proposition 2.9 that $\operatorname{Lip} f\left(x_{0}\right)=\operatorname{Lip}_{A} F\left(x_{0}\right)=\operatorname{Lip} F\left(x_{0}\right)$ and $\operatorname{lip} f\left(x_{0}\right)=\operatorname{lip}_{A} F\left(x_{0}\right)=\operatorname{lip} F\left(x_{0}\right)$. Since $X$ is complete and $A$ is closed it follows that $A$ is locally compact. One can adapt the proof of Proposition 2.9 to show that $\mu$ restricted to $K$ is chunky. Hence, we can now apply Keith's theorem, Theorem 2.8 , to the locally compact set $A$, which completes the proof.

Remark 2.12. Theorem 2.11 is useful even if $(X, d, \mu)$ admits a Poincaré inequality. Indeed, an arbitrary closed, positive measure subset of $X$ need not admit a Poincaré inequality. As an example one can consider the so-called Cantor-diamond set of Koskela and MacManus [19]. This is a connected, compact 2-regular planar set which does not admit any Poincaré inequality. In 
contrast to this, the Lip-lip condition of Theorem 2.8 is inherited by all subsets of $X$ when given the measure $\mu$.

\section{Stepanov's theorem in metric measure spaces}

Theorem 3.1. Let $(X, d, \mu)$ be a metric measure space. Let the measure $\mu$ be a doubling Borel measure. Assume that there is a strong measurable differentiable structure $\left\{\left(X_{\alpha}, \varphi_{\alpha}\right)\right\}$ for $(X, d, \mu)$ with respect to $\operatorname{LIP}(X)$. Then a function $f: X \rightarrow \mathbb{R}$ is $\mu$-a.e. differentiable in $S(f):=\{x \mid \operatorname{Lip} f(x)<\infty\}$ with respect to the structure $\left\{\left(X_{\alpha}, \varphi_{\alpha}\right)\right\}$.

Proof. The proof is based upon Maly's proof of the Euclidean version of Stepanov's theorem, see [20]. By the definition of $S(f)$, for each $x \in S(f)$, there is a neighborhood of $x$ for which $f$ is bounded on. Since $(X, d)$ is separable we can find a countable, dense subset $D$ of $X$. We consider the countable collection of balls $\left\{\mathbf{B}_{j}\right\}$ which have their centers in $D$, rational radius and the property that $f$ is bounded on $\mathbf{B}_{j}$. The definition of $S(f)$ guarantees that it is covered by the union of all such balls. Next we define for every ball $\mathbf{B}_{j}$ two functions $u_{j}: \mathbf{B}_{j} \rightarrow \mathbb{R}$ and $v_{j}: \mathbf{B}_{j} \rightarrow \mathbb{R}$ (the upper and lower Lipschitz envelopes of $f$ ) as follows

$$
\begin{aligned}
& u_{j}(x):=\inf \left\{u(x): u \geq f \text { and } u \text { is } j \text {-Lipschitz on } \mathbf{B}_{j}\right\}, \\
& v_{j}(x):=\sup \left\{v(x): v \leq f \text { and } v \text { is } j \text {-Lipschitz on } \mathbf{B}_{j}\right\} .
\end{aligned}
$$

Since $u_{j}$ is an infimum of $j$-Lipschitz functions that are bounded from below by $f$ which is bounded on $\mathbf{B}_{j}$, we can see that $u_{j}$ is $j$-Lipschitz on $\mathbf{B}_{j}$. Similarly, $v_{j}$ is $j$-Lipschitz on $\mathbf{B}_{j}$ as well.

We now identify the bad subsets of $X$ in terms of $u_{j}$ and $v_{j}$ as follows:

$$
\begin{aligned}
D_{\alpha} & :=\bigcup_{j=1}^{\infty}\left\{x \in \mathbf{B}_{j} \cap X_{\alpha}: u_{j} \text { or } v_{j} \text { is not differentiable at } x\right\} \\
M_{\alpha} & :=\bigcup_{j=1}^{\infty}\left\{x \in \mathbf{B}_{j} \cap X_{\alpha}:\left(u_{j}-v_{j}\right)(x)=0 \text { but } d^{\alpha}\left(u_{j}-v_{j}\right)(x) \neq 0\right\} \\
N & :=\bigcup_{\alpha}\left(D_{\alpha} \cup M_{\alpha}\right) .
\end{aligned}
$$

Note that for each $\alpha$, the set $D_{\alpha}$ is by Definition 2.3 a null set and the same holds for $M_{\alpha}$ by Corollary 2.10. Thus, $N$ is a countable union of null sets, i.e., $N$ has measure zero as well.

Take a point $x_{0} \in S(f) \backslash N$ and choose $\alpha$ so that $x_{0} \in X_{\alpha}$. Since Lip $f\left(x_{0}\right)$ is finite, there exist $r>0$ and $l>0$ such that

$$
\left|f(x)-f\left(x_{0}\right)\right| \leq l \cdot d\left(x, x_{0}\right) \text { for all } x \in \mathbf{B}\left(x_{0}, r\right)
$$

Using the separability of $X$ we can find an element $\mathbf{B}_{j}$ of the sequence $\left\{\mathbf{B}_{j}\right\}$ with $j>l$ satisfying

$$
x_{0} \in \mathbf{B}_{j} \subset \mathbf{B}\left(x_{0}, r\right) .
$$

It follows that for $x \in \mathbf{B}_{j}$ we have

$$
f\left(x_{0}\right)-j d\left(x, x_{0}\right) \leq f(x) \leq f\left(x_{0}\right)+j d\left(x, x_{0}\right) .
$$


By the definition of $u_{j}$ and $v_{j}$ we obtain that for each $x \in \mathbf{B}_{j}$

$$
f\left(x_{0}\right)-j d\left(x, x_{0}\right) \leq v_{j}(x) \leq f(x) \leq u_{j}(x) \leq f\left(x_{0}\right)+j d\left(x, x_{0}\right)
$$

in particular, $u_{j}\left(x_{0}\right)=f\left(x_{0}\right)=v_{j}\left(x_{0}\right)$. Since $x_{0} \notin M_{\alpha}$ we know that $d^{\alpha} v_{j}\left(x_{0}\right)=d^{\alpha} u_{j}\left(x_{0}\right)$. Using (3.1), we build the chain of inequalities

$$
\begin{aligned}
0 & =\lim _{\substack{x \rightarrow x_{0} \\
x \neq x_{0}}} \frac{v_{j}(x)-v_{j}\left(x_{0}\right)-\left\langle d^{\alpha} v_{j}\left(x_{0}\right), \varphi_{\alpha}(x)-\varphi_{\alpha}\left(x_{0}\right)\right\rangle}{d\left(x, x_{0}\right)} \\
& \leq \lim _{\substack{x \rightarrow x_{0} \\
x \neq x_{0}}} \frac{f(x)-f\left(x_{0}\right)-\left\langle d^{\alpha} u_{j}\left(x_{0}\right), \varphi_{\alpha}(x)-\varphi_{\alpha}\left(x_{0}\right)\right\rangle}{d\left(x, x_{0}\right)} \\
& \leq \lim _{\substack{x \rightarrow x_{0} \\
x \neq x_{0}}} \frac{u_{j}(x)-u_{j}\left(x_{0}\right)-\left\langle d^{\alpha} u_{j}\left(x_{0}\right), \varphi_{\alpha}(x)-\varphi_{\alpha}\left(x_{0}\right)\right\rangle}{d\left(x, x_{0}\right)}=0 .
\end{aligned}
$$

In conclusion

$$
\lim _{\substack{x \rightarrow x_{0} \\ x \neq x_{0}}} \frac{f(x)-f\left(x_{0}\right)-\left\langle d^{\alpha} v_{j}\left(x_{0}\right), \varphi_{\alpha}(x)-\varphi_{\alpha}\left(x_{0}\right)\right\rangle}{d\left(x, x_{0}\right)}=0 .
$$

Since $d^{\alpha} f=d^{\alpha} u_{j} \mu$-a.e. in $\mathbf{B}_{j}$ and the balls $\left\{\mathbf{B}_{j}\right\}_{j}$ cover the whole set $S(f) \backslash N$ the uniqueness and the measurability of $d^{\alpha} f$ follow.

Remark 3.2. Motivated by Keith's Lip-lip condition from Theorem 2.8 it is natural to ask whether a function $f: X \rightarrow \mathbb{R}$ is differentiable $\mu$-a.e. in the set

$$
s(f)=\{x \in X: \operatorname{lip} f(x)<\infty\} .
$$

Examples constructed in [3] show that the answer to this question is negative already for $X=$ $[0,1]$. However, the a.e. differentiability holds true for functions in $\mathbb{R}^{n}$ if we assume that $f$ is continuous, lip $f$ is finite outside a set of $\sigma$-finite Hausdorff $n-1$ measure and lip $f \in L^{p}$ for $p>n$.

\section{Applications}

The first part of following statement follows from Theorem 5.1 in [15]. Its proof uses Riesz potentials and maximal functions. For the convenience of the reader we present a direct proof, based on the method of ball-chaining which has been employed in $[14,15,21]$.

Theorem 4.1. Let $(X, d, \mu)$ be a doubling metric measure space. Let $f: X \rightarrow \mathbb{R}$ be a measurable function and $\rho \in L_{\text {loc }}^{p}$ with $p>Q$ and $p \geq 1$ where $Q$ is as in (2.4). If $(f, \rho)$ is a pair which satisfies $(1, p)$-Poincaré-inequality, then $f$ has a representative which is locally $(1-Q / p)$-Hölder continuous which is $\mu$-a.e. differentiable with respect to the strong measurable differentiable structure $\left\{\left(X_{\alpha}, \varphi_{\alpha}\right)\right\}$ whenever $X$ admits such structure on all of LIP $(X)$.

Proof. Let $z \in X, R>0$ and $x \in \mathbf{B}(z, R / 2)$ be a Lebesgue point of $f$. Let $\mathbf{B}_{0}=\mathbf{B}(z, R)$ and for $i \in \mathbb{N}$, let $\mathbf{B}_{i}=\mathbf{B}\left(x, R / 4^{i}\right)$. Then for each $i \in \mathbb{N}, \mathbf{B}_{i} \subset \mathbf{B}_{i-1}$ and $\operatorname{rad} \mathbf{B}_{i}=\frac{1}{4} \operatorname{rad} \mathbf{B}_{i-1}$. Since $x$ is a Lebesgue point of $f$

$$
f(x)=\lim _{i \rightarrow \infty} f_{\mathbf{B}_{i}} f d \mu
$$


In the following chain of estimates $C>0$ is a generic constant whose value can vary from line to line.

$$
\begin{aligned}
\left|f(x)-f_{\mathbf{B}_{0}}\right| & \leq \sum_{i=0}^{\infty}\left|f_{\mathbf{B}_{i}}-f_{\mathbf{B}_{i+1}}\right| \\
& \leq \sum_{i=0}^{\infty} f_{\mathbf{B}_{i}}\left|f-f_{\mathbf{B}_{i}}\right| \\
& \leq C \sum_{i=0}^{\infty} \frac{R}{4^{i}}\left(f_{\tau \mathbf{B}_{i}} \rho^{p}\right)^{1 / p} \quad \text { (Poincaré-inequality) } \\
& \leq C \sum_{i=0}^{\infty} \frac{R}{4^{i}}\left(\frac{\mu\left(\mathbf{B}_{0}\right)}{\mu\left(\mathbf{B}_{i}\right)}\right)^{1 / p} \cdot\left(f_{\tau \mathbf{B}_{0}} \rho^{p}\right)^{1 / p} \\
& \leq C R \sum_{i=0}^{\infty}\left(\frac{1}{4^{i}}\right)^{1-Q / p}\left(\int_{\tau \mathbf{B}_{0}}{ }^{p}\right)^{1 / p} \quad \text { (by (2.4)) } \\
& \leq C R\left(\int_{\tau \mathbf{B}_{0}} \rho^{p}\right)^{1 / p} \sum_{i=0}^{\infty}\left(\frac{1}{4^{i}}\right)^{1-Q / p} \\
& =C R\left(f_{\tau \mathbf{B}_{0}} \rho^{p}\right)^{1 / p} .
\end{aligned}
$$

Hence, for Lebesgue points $x, y \in \mathbf{B}(z, R / 2)$ we have that

$$
|f(x)-f(y)| \leq C R\left(\frac{1}{\mu(\mathbf{B}(z, \tau R))}\right)^{1 / p}\left(\int_{\mathbf{B}(z, \tau R)} \rho^{p}\right)^{1 / p} .
$$

Fix $p \in X$ and let $r<\operatorname{diam} X$. Then for $x, y \in \mathbf{B}\left(p, \frac{1}{4} r\right)$ we have that $\mathbf{B}(x, 2 d(x, y)) \subseteq$ $\mathbf{B}(p, r)$. By setting $z=x$ and $R=2 d(x, y)$ we see that for each pair of Lebesgue points $x$ and $y$ in $\mathbf{B}\left(p, \frac{1}{4} r\right)$, that

$$
|f(x)-f(y)| \leq C d(x, y)\left(\frac{1}{\mu(\mathbf{B}(x, 2 \tau d(x, y)))}\right)^{1 / p}\left(\int_{\mathbf{B}(p, \tau r)} \rho^{p} d \mu\right)^{1 / p} .
$$

Using (2.4) allows us to conclude that for each pair of Lebesgue points $x$ and $y$ in the ball $\mathbf{B}(p, r / 4)$ that

$$
|f(x)-f(y)| \leq C d(x, y)^{1-Q / p}\left(f_{\mathbf{B}(p, \tau r)} \rho^{p} d \mu\right)^{1 / p}
$$

which implies that $f$ is locally Hölder continuous on the set of Lebesgue points of $f$. Hence, there exists a representative of $f$ which is locally $(1-Q / p)$-Hölder continuous. By choosing this representative, every point is a Lebesgue point of $f$. Now using this representative of $f$, we will show that $\operatorname{Lip} f(x)$ is finite for $\mu$-a.e. $x$.

Indeed, for each $x \in X$, and $R>0$ we have by (4.1) that

$$
\sup _{y \in \mathbf{B}(x, R)}\left(\frac{|f(x)-f(y)|}{d(x, y)}\right) \leq C\left(f_{\mathbf{B}(x, 2 \tau R)} \rho^{p} d \mu\right)^{1 / p}
$$


which implies that

$$
\operatorname{Lip} f(x) \leq C \limsup _{R \rightarrow 0}\left(f_{\mathbf{B}(x, 2 \tau R)} \rho^{p} d \mu\right)^{1 / p}
$$

which is finite whenever $x$ is a Lebesgue point of $\rho$. Hence, for $\mu$-a.e. $x, \operatorname{Lip} f(x)$ is finite. By Theorem 3.1 the proof is complete.

In the following we will place the above theorem in the context of Sobolev spaces defined on metric measure spaces. The interested reader is encouraged to consult the overview article [11] by Hajłasz for an introduction to Sobolev spaces in metric measure spaces. The interested reader should also consult [10] for another approach to creating Sobolev spaces in abstract metric measure spaces.

For the record we first recall the notion Newtonian space (as introduced by N. Shanmugalingam in [21]).

Definition 4.2. Let $(X, d, \mu)$ be a metric measure space with $\mu$ doubling that satisfies a $(1, p)$ Poincaré inequality. We define the vector space of functions, $\tilde{N}^{1, p}$ as the set of all $p$-integrable functions $f$ so that there exists a non negative $p$-integrable Borel function $\rho$ which is a weak $p$-uppergradient of $f$, i.e., for $\bmod _{p}$-a.e. curve $\gamma:[0, T] \rightarrow X$,

$$
|f(\gamma(T))-f(\gamma(0))| \leq \int_{\gamma} \rho d \mathcal{H}^{1}
$$

where $\mathcal{H}^{1}$ is the 1 -Hausdorff measure. Here, $\bmod _{p}(\Gamma)$ of a curve family $\Gamma$ is defined as

$$
\bmod _{p}(\Gamma):=\inf \left\{\int \rho^{p} d \mu \mid \text { for each curve } \gamma \in \Gamma, \int_{\gamma} \rho d \mathcal{H}^{1} \geq 1\right\}
$$

The Newtonian space $N^{1, p}(X)$ is the set of equivalence classes of the relation $f \sim g$ iff $\|f-g\|_{N^{1, p}}=0$ where

$$
\|f\|_{N^{1, p}}:=\|f\|_{p}+\inf \left\{\|\rho\|_{p} \mid \rho \text { is a weak } p \text {-uppergradient of } f\right\} .
$$

We also define $N_{\text {loc }}^{1, p}(X)$ as the space of functions in $L_{\text {loc }}^{p}$ which have a weak $p$-uppergradient $\rho \in L_{\text {loc }}^{p}$.

Since $N^{1, p}(X)=W^{1, p}\left(\mathbb{R}^{n}\right)$ when $X=\mathbb{R}^{n}$, the following statement is a direct generalization of Calderon's theorem.

Corollary 4.3. Let $\mu$ be a doubling measure that satisfies a $(1, p)$-Poincare inequality with $p \geq 1$. If $p>Q$ where $Q$ is the exponent as in (2.4), then each function $f \in N^{1, p}(X)$ has a locally Hölder continuous representative which is differentiable $\mu$-a.e.

Proof. Let $f \in N^{1, p}(X)$ and $\rho$ be a weak $p$-upper gradient of $f$. Then the pair $(f, \rho)$ satisfies the Poincaré inequality, see [21]. Apply Theorem 4.1 to finish.

In $\mathbb{R}^{n}$, Calderon's Theorem gives that if $f \in W^{1, p}\left(\mathbb{R}^{n}\right)$ with $p>n$, then $f$ is differentiable a.e. and the pointwise gradient of $f$ is equal to the weak gradient of $f$ a.e. In the following we will show that when one extends the differential operator $d$ to $N^{1, p}$, then the extension $D$ agrees with the $d$ as calculated by Theorem 3.1. In the following we first show how to use a result of 
Franchi, Hajłasz, and Koskela to extend $d$ to $N^{1, p}$. This extension is the only extension which is stable under limits in $N^{1, p}$.

Let $(X, d, \mu)$ be a metric measure space for which $\mu$ is doubling that satisfies a Poincaré inequality. Fix a strong measurable differentiable structure on $X,\left\{\left(X_{\alpha}, \varphi_{\alpha}\right)\right\}$ created by Theorem 2.6. For $\mu$-a.e. $x$, let $\alpha(x)$ denote the unique $\alpha$ so that $x \in X_{\alpha}$. By [5, Theorem 4.38], for each $x \in X_{\alpha}$, the semi-norm

$$
\|\lambda\|_{x}=\operatorname{Lip}\left(\left\langle\lambda, \varphi_{\alpha}\right\rangle\right)(x)
$$

is a norm on $\mathbb{R}^{N(\alpha)}$. We see that for each $\lambda \in \mathbb{R}^{N(\alpha(x))}$

$$
\begin{aligned}
\|\lambda\|_{x} & =\operatorname{Lip}\left(\left\langle\lambda, \varphi_{\alpha}\right\rangle\right)(x) \\
& \leq\|\lambda\|_{\mathbb{R}^{N(\alpha)}} \cdot \operatorname{LIP}\left(\varphi_{\alpha}\right)
\end{aligned}
$$

where $\|\cdot\|_{\mathbb{R}^{N(\alpha)}}$ is the Euclidean norm on $\mathbb{R}^{N(\alpha)}$. Letting $C_{\alpha}=\operatorname{LIP}\left(\varphi_{\alpha}\right)$ we see that for $\mu$-a.e. $x \in X$,

$$
\|\lambda\|_{x} \leq C_{\alpha(x)}\|\lambda\|_{\mathbb{R}^{N(\alpha(x))}} .
$$

Moreover, since $\|\cdot\|_{x}$ is a norm on $\mathbb{R}^{N(\alpha)}$, for each $x \in X_{\alpha}$ there exists a constant $C(x)$ so that

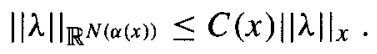

By partitioning $X_{\alpha}$ into

$$
U_{\alpha, n}=\left\{x \in X_{\alpha} \mid n \geq C(x)>n-1\right\}
$$

we have that for each $x \in U_{\alpha, n}$

$$
\frac{1}{n}\|\lambda\|_{\mathbb{R}^{N(\alpha(x))}} \leq\|\lambda\|_{x} \leq n\|\lambda\|_{\mathbb{R}^{N(\alpha(x))}} .
$$

For each locally Lipschitz function $u, d^{\alpha} u$ is measurable and so is the function $x \rightarrow \operatorname{Lip}(u)(x)$. Let $u$ be differentiable at $x_{0} \in X_{\alpha}$ and set $f(x)=\left\langle d^{\alpha} u\left(x_{0}\right), \varphi_{\alpha}(x)-\varphi_{\alpha}\left(x_{0}\right)\right\rangle$. The definition of differentiability of $u$ at $x_{0}$ gives that $\operatorname{Lip}(u-f)\left(x_{0}\right)=0$. Hence, we see that

$$
\begin{aligned}
\operatorname{Lip}(u)\left(x_{0}\right) & \leq \operatorname{Lip}(u-f)\left(x_{0}\right)+\operatorname{Lip}(f)\left(x_{0}\right) \\
& =0+\left\|d^{\alpha} u\left(x_{0}\right)\right\|_{x_{0}}
\end{aligned}
$$

and

$$
\begin{aligned}
\left\|d^{\alpha} u\left(x_{0}\right)\right\|_{x_{0}} & =\operatorname{Lip}(f)\left(x_{0}\right) \\
& \leq \operatorname{Lip}(f-u)\left(x_{0}\right)+\operatorname{Lip}(u)\left(x_{0}\right) \\
& =\operatorname{Lip}(u)\left(x_{0}\right)
\end{aligned}
$$

i.e., $\operatorname{Lip}(u)\left(x_{0}\right)=\left\|d^{\alpha} u\left(x_{0}\right)\right\|_{x_{0}}$ whenever $u$ is differentiable at $x_{0} \in X_{\alpha}$. To simplify notation we will write $d u(x)$ for $d^{\alpha(x)} u(x)$. The above implies that for each locally Lipschitz function $u$, $x \rightarrow\|d u(x)\|_{x}$ is measurable. Moreover, from the algebraic nature of Equation (2.1) we see that for each pair of locally Lipschitz functions $u$ and $v$, that $d(u v)(x)=v(x) d u(x)+u(x) d v(x)$. Together with Proposition 2.9 we see that the mapping $d$ satisfies the following:

(1) $\|d u(x)\|_{x}=\operatorname{Lip}(u)(x) \leq \operatorname{LIP}(u)$ provided that $d u(x)$ exists.

(2) For each $\lambda \in \mathbb{R}, d(\lambda u)(x)=\lambda d u(x)$ provided that $d u(x)$ exists.

(3) $d(u+v)(x)=d u(x)+d v(x)$ provided that both $d u(x)$ and $d v(x)$ exist. 
(4) $\quad d(u v)(x)=v(x) d u(x)+u(x) d v(x)$ provided that both $d u(x)$ and $d v(x)$ exist.

Now let $N=\sup _{\alpha} N(\alpha)$, which is finite by Cheeger's theorem, Theorem 2.6. By Keith [18, Lemma 6.10 and 6.16], for $\mu$-a.e. $x \in X_{\alpha}$, there exists an inner product on $\mathbb{R}^{N(\alpha)},\langle\cdot, \cdot\rangle_{x}$ so that for each $v \in \mathbb{R}^{N(\alpha)}$,

$$
\frac{1}{C(N)}\|v\|_{x} \leq\langle v, v\rangle_{x}^{1 / 2} \leq C(N)\|v\|_{x} .
$$

In contrast to (4.3), $C(N)$ does not depend on $\alpha$. Moreover, the mapping $x \rightarrow\langle\cdot, \cdot\rangle_{x}$ is measurable as a map from $X_{\alpha}$ to the space of positive definite matrices. For each $x \in X_{\alpha}$, let $e_{x}^{1}, \ldots, e_{x}^{N(\alpha)}$ be an orthonormal basis of $\mathbb{R}^{N(\alpha)}$ with respect to the inner product $\langle\cdot, \cdot\rangle_{x}$ so that the functions $x \rightarrow e_{x}^{i}$ are measurable (such a choice exists because the mapping $x \rightarrow\langle\cdot, \cdot\rangle_{x}$ is measurable). Writing $d u(x)=\sum_{i} a_{i}(x) e_{x}^{i}$, we see that the function $\widetilde{d} u(x)=\left(a_{1}(x), a_{2}(x), \ldots\right)$ is a measurable function which satisfies the following.

(1) $\|\widetilde{d} u(x)\| \leq C\|d u(x)\|_{x} \leq C \operatorname{LIP}(u)(x)$ where $\|\cdot\|$ is the Euclidean norm on $\mathbb{R}^{N(\alpha)}$.

(2) For each $\lambda \in \mathbb{R}, \widetilde{d}(\lambda u)(x)=\lambda \widetilde{d} u(x)$.

(3) $\tilde{d}(u+v)(x)=\tilde{d} u(x)+\tilde{d} v(x)$.

(4) $\tilde{d}(u v)(x)=v(x) \tilde{d} u(x)+u(x) \tilde{d} v(x)$.

(5) The pair $(u,\|\widetilde{d} u\|)$ satisfies a $(1, p)$-Poincaré inequality with constants independent of $u$, i.e., there exists constants $C>0$ and $\tau \geq 1$ so that for each locally Lipschitz function $u$, and for each ball $\mathbf{B}$,

$$
f_{\mathbf{B}}\left|u-u_{\mathbf{B}}\right| d \mu \leq C \operatorname{diam}(\mathbf{B})\left(f_{\tau \mathbf{B}}\|\widetilde{d} u\|^{p} d \mu\right)^{1 / p} .
$$

Now if we view $\widetilde{d} u(x)$ as element of $\mathbb{R}^{N}$, via

$$
\widetilde{d} u(x)=\left(a_{1}(x), \ldots, a_{N(\alpha)}(x), 0, \ldots, 0\right) \in \mathbb{R}^{N(\alpha(x))} \times \mathbb{R}^{N-N(\alpha(x))}
$$

we then have produced a function $\widetilde{d}$ which maps the space of locally Lipschitz functions to $\mathbb{R}^{N}$ so that (1)-(5) from above are true. We now extend $\widetilde{d}$ to $N^{1, p}$ as follows.

In [9], Franchi, Hajlasz, and Koskela showed that if a metric measure space $(X, d, \mu)$ admits a Poincare inequality with $\mu$ doubling and if there is a function $\widetilde{d}$ as above acting on the space of locally Lipschitz function, then $\widetilde{d}$ extends uniquely to all of $N^{1, p}(X)$ as follows: if $f_{n}$ is a collection of locally Lipschitz functions so that $f_{n} \rightarrow f$ in $N^{1, p}(X)$, then there exists a measurable function $v$ with $v(x) \in \mathbb{R}^{N(\alpha(x))}$ so that $h_{n}(x)=\left\|\tilde{d} f_{n}(x)-v(x)\right\|$ converges to 0 in $L^{p}(X)$ and for any other sequence of locally Lipschitz functions $g_{n}$ that converges to $f$ in $N^{1, p}(X)$ satisfies $\widetilde{d} g_{n} \rightarrow v$ in $L^{p}$ as well. We will write $\widetilde{D} f$ for this $v$. In particular, if $u$ is locally Lipschitz, then $\widetilde{d} u(x)=\widetilde{D} u(x)$ for $\mu$-a.e. $x$. By passing to limits we see that the same properties of $\widetilde{d}$ are inherited by $\widetilde{D}$, i.e., for each pair of function $u, v \in N^{1, p}(X)$ and $\mu$-a.e. $x$

(1) For each $\lambda \in \mathbb{R}, \tilde{D}(\lambda u)(x)=\lambda D u(x)$.

(2) $\widetilde{D}(u+v)(x)=\widetilde{D} u(x)+\widetilde{D} v(x)$.

(3) $\widetilde{D}(u v)(x)=v(x) \widetilde{D} u(x)+u(x) \widetilde{D} v(x)$ provided that $v$ is bounded and Lipschitz.

Moreover, we can extend $\widetilde{D}$ to the space $N_{\text {loc }}^{1, p}$ by multiplying such functions by Lipschitz bump functions. Note that if $N(\alpha(x))<N$, then for each $u \in N^{1, p}$ and $i>N(\alpha(x))$, the $i$-th co-ordinate of $\widetilde{D} u(x)$ is zero. We now define $D u(x) \in \mathbb{R}^{N(\alpha)}$ as

$$
D u(x)=\sum_{i} a_{i}(x) e_{x}^{i}
$$


where $\widetilde{D} u(x)=\left(a_{1}(x), a_{2}(x), \ldots\right)$. In this fashion we have extended $d$ from the space of locally Lipschitz functions to $D$ acting on $N^{1, p}(X)$ so that $d=D$ on the space of locally Lipschitz functions so that if $f_{n} \rightarrow f$ in $\mathrm{N}^{1, p}$ then $\int\left\|D f_{n}(x)-D f(x)\right\|_{x}^{p} \rightarrow 0$.

Theorem 4.4. Let $(X, d, \mu)$ be a metric measure space with $\mu$ doubling that admits a $(1, p)$ Poincaré inequality. If $f \in N^{1, p}(X)$ with $p>Q$ where $Q$ as in (2.4) then $d f(x)=D f(x)$ $\mu$-a.e. where $D$ is the unique extension of $d$ to $N^{1, p}(X)$.

Proof. Fix a strong measurable differentiable structure $\left\{\left(\varphi_{\alpha}, X_{\alpha}\right)\right\}_{\alpha \in A}$ on $X$. By inequality (4.3) and possibly reindexing the charts $X_{\alpha, n}$ to $X_{\alpha}$, we can assume that for each $\alpha$ there exists $C_{\alpha}>0$ so that for each $\lambda \in \mathbb{R}^{N(\alpha)}$ and each $x \in X_{\alpha}$

$$
\frac{1}{C_{\alpha}}\|\lambda\|_{\mathbb{R}^{N(\alpha)}} \leq\|\lambda\|_{x} \leq C_{\alpha}\|\lambda\|_{\mathbb{R}^{N(\alpha)}}
$$

where $\|\cdot\|_{\mathbb{R}^{N(\alpha)}}$ is the Euclidean norm on $\mathbb{R}^{N(\alpha)}$. Let $f_{n}$ be a sequence of locally Lipschitz functions that converge to $f$ in $N^{1, p}(X)$, i.e.,

$$
\int_{X}\left\|d f_{n}(x)-D f(x)\right\|_{x}^{p} d \mu(x) \rightarrow 0
$$

and

$$
\int_{X}\left|f_{n}(x)-f(x)\right|^{p} d \mu(x) \rightarrow 0 .
$$

For each $n, \rho_{n}(x)=\left\|D f_{n}(x)\right\|_{x}$ is a weak $p$-upper gradient of $f_{n}$. Letting $\rho(x)=\|D f(x)\|_{x}$ we see that since $f_{n} \rightarrow f$ and $\rho_{n} \rightarrow \rho$ in $L^{p}$, the pair $(f, \rho)$ satisfies a $(1, \mathrm{p})$-Poincaré inequality. Since $x \rightarrow\|D f(x)\|_{x}$ is $L^{p}$ integrable we see that for $\mu$-a.e. $x_{0} \in X_{\alpha}$

$$
\limsup _{r \rightarrow 0} \frac{1}{\mu\left(\mathbf{B}\left(x_{0}, r\right)\right)} \int_{\mathbf{B}\left(x_{0}, r\right) \backslash X_{\alpha}}\|D f(y)\|_{y}^{p} d \mu(y)=0 .
$$

For each $\alpha$ and $x \in X_{\alpha}$ let $D^{\alpha} f(x)=D f(x)$ which is an element in $\mathbb{R}^{N(\alpha)}$. We can view the map $x \rightarrow D^{\alpha} f(x)$ as a function from $X_{\alpha}$ to $\mathbb{R}^{N(\alpha)}$. Extend $D^{\alpha} f$ as zero outside of $X_{\alpha}$. We now define a good subset of $X_{\alpha}$ as

$$
\begin{aligned}
S_{\alpha}=\left\{x_{0} \in X_{\alpha} \mid\right. & x_{0} \text { is a Lebesgue point of } D^{\alpha} f, \\
& \left.x_{0} \text { is a point of density of } X_{\alpha} \text { and (4.5) holds }\right\} .
\end{aligned}
$$

Note that $\mu\left(X_{\alpha} \backslash S_{\alpha}\right)=0$. For each $x_{0} \in S_{\alpha}$, consider the function $g$ defined by

$$
g(x)=f(x)-f\left(x_{0}\right)-\left\langle\lambda, \varphi_{\alpha}(x)-\varphi_{\alpha}\left(x_{0}\right)\right\rangle
$$

where $\lambda=D f\left(x_{0}\right)$ viewing $D f\left(x_{0}\right)$ as an element of $\mathbb{R}^{N(\alpha)}$. Note that for each ball $\mathbf{B}, g \in$ $N^{1, p}(\mathbf{B})$. One sees that

$$
\begin{aligned}
D g(x) & =D f(x)-D\left(\left\langle\lambda, \varphi_{\alpha}\right\rangle\right)(x) \\
& =D f(x)-\sum_{i=1}^{N(\alpha)} \lambda_{i} D \varphi_{\alpha}^{i}(x) \\
& =D f(x)-\sum_{i=1}^{N(\alpha)} \lambda_{i} d \varphi_{\alpha}^{i}(x)
\end{aligned}
$$


Moreover, for $x \in X_{\alpha}, d \varphi_{\alpha}^{i}=e_{i}$ where $e_{i}$ is the vector in $\mathbb{R}^{N(\alpha)}$ whose $i$-th co-ordinate is one and all others are zero. In particular,

$$
D g(x)=D f(x)-D f\left(x_{0}\right)
$$

for $x \in X_{\alpha}$. Since $f_{n} \rightarrow f$ in $N^{1, p}$, we see that for each ball $\mathbf{B}, g_{n} \rightarrow g$ in $N^{1, p}(\mathbf{B})$ where

$$
g_{n}(x)=f_{n}(x)-f\left(x_{0}\right)-\left\langle\lambda, \varphi_{\alpha}(x)-\varphi_{\alpha}\left(x_{0}\right)\right\rangle .
$$

Hence, by a limiting argument, the pair $\left(g, x \rightarrow\|D g(x)\|_{x}\right)$ satisfies a $(1, p)$-Poincaré inequality. In particular, for each $r>0$ and $x \in \mathbf{B}\left(x_{0}, r\right)$, inequality (4.2) gives

$$
\frac{\left|g(x)-g\left(x_{0}\right)\right|}{d\left(x, x_{0}\right)} \leq C\left(f_{\mathbf{B}\left(x_{0}, 2 \tau r\right)}\left\|D f(y)-\sum_{i=1}^{N(\alpha)} \lambda_{i} d \varphi_{\alpha}^{i}(y)\right\|_{y}^{p} d \mu(y)\right)^{1 / p}
$$

We claim that

$$
\underset{r \rightarrow 0}{\limsup }\left(f_{\mathbf{B}\left(x_{0}, r\right)}\left\|D f(y)-\sum_{i=1}^{N(\alpha)} \lambda_{i} d \varphi_{\alpha}^{i}(y)\right\|_{y}^{p} d \mu(y)\right)^{1 / p}=0 .
$$

Indeed, since $x_{0}$ is a Lebesgue point of $D^{\alpha} f$ and (4.4) we see that

$$
\begin{aligned}
& \limsup _{r \rightarrow 0}\left(\frac{1}{\mu\left(\mathbf{B}\left(x_{0}, r\right)\right)} \int_{\mathbf{B}\left(x_{0}, r\right) \cap X_{\alpha}}\left\|D f(y)-\sum_{i=1}^{N(\alpha)} \lambda_{i} d \varphi_{\alpha}^{i}\right\|_{y}^{p} d \mu(y)\right)^{1 / p} \\
& \quad=\limsup _{r \rightarrow 0}\left(\frac{1}{\mu\left(\mathbf{B}\left(x_{0}, r\right)\right)} \int_{\mathbf{B}\left(x_{0}, r\right) \cap X_{\alpha}}\left\|D^{\alpha} f(y)-D^{\alpha} f\left(x_{0}\right)\right\|_{y}^{p} d \mu(y)\right)^{1 / p} \\
& \quad \leq \limsup _{r \rightarrow 0} C_{\alpha}\left(\frac{1}{\mu\left(\mathbf{B}\left(x_{0}, r\right)\right)} \int_{\mathbf{B}\left(x_{0}, r\right) \cap X_{\alpha}}\left\|D^{\alpha} f(y)-D^{\alpha} f\left(x_{0}\right)\right\|_{\mathbb{R}^{N(\alpha)}}^{p} d \mu(y)\right)^{1 / p} \\
& \quad \leq \limsup _{r \rightarrow 0} C_{\alpha}\left(f_{\mathbf{B}\left(x_{0}, r\right)}\left\|D^{\alpha} f(y)-D^{\alpha} f\left(x_{0}\right)\right\|_{\mathbb{R}^{N(\alpha)}}^{p} d \mu(y)\right)^{1 / p}=0 .
\end{aligned}
$$

Since (4.5) holds and $x_{0}$ is a point of density of $X_{\alpha}$,

$$
\begin{aligned}
& \limsup _{r \rightarrow 0}\left(\frac{1}{\mu\left(\mathbf{B}\left(x_{0}, r\right)\right)} \int_{\mathbf{B}\left(x_{0}, r\right) \backslash X_{\alpha}}\left\|D f(y)-\sum_{i=1}^{N(\alpha)} \lambda_{i} d \varphi_{\alpha}^{i}\right\|_{y}^{P} d \mu(y)\right)^{1 / p} \\
& \leq \limsup _{r \rightarrow 0}\left(\frac{1}{\mu\left(\mathbf{B}\left(x_{0}, r\right)\right)} \int_{\mathbf{B}\left(x_{0}, r\right) \backslash X_{\alpha}}\|D f(y)\|_{y}^{p} d \mu(y)\right)^{1 / p} \\
& \quad+\operatorname{LIP}\left(\varphi_{\alpha}\right) \cdot\|\lambda\|_{\mathbb{R}^{N(\alpha)}} \cdot \limsup _{r \rightarrow 0}\left(\frac{\mu\left(\mathbf{B}\left(x_{0}, r\right) \backslash X_{\alpha}\right)}{\mu\left(\mathbf{B}\left(x_{0}, r\right)\right)}\right)^{1 / p}=0 .
\end{aligned}
$$

Thus, for $\lambda=D f\left(x_{0}\right)$

$$
\begin{aligned}
0 & =\limsup _{x \rightarrow x_{0}} \frac{\left|g(x)-g\left(x_{0}\right)\right|}{d\left(x, x_{0}\right)} \\
& =\limsup _{x \rightarrow x_{0}} \frac{\left|f(x)-f\left(x_{0}\right)-\left\langle\lambda, \varphi_{\alpha}(x)-\varphi_{\alpha}\left(x_{0}\right)\right\rangle\right|}{d\left(x, x_{0}\right)}
\end{aligned}
$$


which by uniqueness property of the strong differentiable structure of $\left\{\left(X_{\alpha}, \varphi_{\alpha}\right)\right\}$ implies that $d f\left(x_{0}\right)=\lambda=D f\left(x_{0}\right)$, completing the proof.

Remark 4.5. With the additional hypothesis that $X$ is complete, $D f$ can be shown to be an integral average pointwise differential, even if $p \leq Q$. Indeed, Björn in [4] has shown that if $(X, d, \mu)$ is a complete metric measure space with $\mu$ doubling and admits a $(1, p)$-Poincaré inequality, then for each $f \in N^{1, p}(X)$ and $\mu$-a.e. $x_{0} \in X$,

$$
\limsup _{r \rightarrow 0} \frac{1}{r} f_{\mathbf{B}\left(x_{0}, r\right)}\left|f(x)-f\left(x_{0}\right)-\left\langle\lambda, \varphi_{\alpha\left(x_{0}\right)}(x)-\varphi_{\alpha\left(x_{0}\right)}\left(x_{0}\right)\right\rangle\right| d \mu(x)=0
$$

where $\lambda=D f\left(x_{0}\right)$.

Along similar lines, Keith in [17] has also shown that if $(X, d, \mu)$ is a complete metric measure space with $\mu$ doubling and admits a $(1, p)$-Poincaré inequality, then for each $f \in N^{1, p}(X)$ and $\mu$-a.e. $x_{0} \in X, D f$ is an approximate differential of $f$ at $x_{0}$ i.e.,

$$
\operatorname{aplim}_{x \rightarrow x_{0}} \frac{\left|f(x)-f\left(x_{0}\right)-\left\langle D f\left(x_{0}\right), \varphi_{\alpha\left(x_{0}\right)}(x)-\varphi_{\alpha\left(x_{0}\right)}\left(x_{0}\right)\right\rangle\right|}{d\left(x, x_{0}\right)}=0
$$

where $L=\operatorname{aplim}_{y \rightarrow x} u(x)$ if and only if for each $\epsilon>0$,

$$
\lim _{r \rightarrow 0} \frac{\mu(\{y \in \mathbf{B}(x, r):|L-u(y)|>\epsilon\})}{\mu(\mathbf{B}(x, r))}=0 .
$$

Theorem 4.1 replaces $\operatorname{aplim}_{x \rightarrow x_{0}}$ of the above with $\lim _{x \rightarrow x_{0}}$ when $p>Q$.

A weaker notion of differentiability is $L^{q}$-differentiability. Namely a function $f$ is $L^{q}$ differentiable at a point $x_{0}$ if there exists $\lambda \in \mathbb{R}^{N\left(\alpha\left(x_{0}\right)\right)}$ so that

$$
\limsup _{r \rightarrow 0} \frac{1}{r}\left(f_{\mathbf{B}\left(x_{0}, r\right)}\left|f(x)-f\left(x_{0}\right)-\left(\lambda, \varphi_{\alpha\left(x_{0}\right)}(x)-\varphi_{\alpha\left(x_{0}\right)}\left(x_{0}\right)\right\rangle\right|^{q} d \mu(x)\right)^{1 / q}=0 .
$$

Note that if $f$ is differentiable at $x_{0}$ then for each $1 \leq q<\infty, f$ is $L^{q}$-differentiable at $x_{0}$ with $\lambda=D f\left(x_{0}\right)$. One can readily see that (4.6) states that each $f \in N^{1, p}$ is $L^{1}$-differentiable at $\mu$-a.e. $x_{0} \in X$. From [22, Theorem VIII.1], when $X=\mathbb{R}^{n}$ with the Euclidean metric and $\mu$ is the Lebesgue measure, for $f \in W^{1, p}\left(\mathbb{R}^{n}\right)$ with $1<p<n, f$ is $L^{p^{*}}$-differentiable a.e. where $p^{*}=\frac{p n}{n-p}$ (the Sobolev conjugate of $p$ ). For finer notions of the degree of smoothness of a function we refer the reader to [6].

In the case when the function has bounded variation, $f \in \mathrm{BV}\left(\mathbb{R}^{n}\right)$ it follows that $f$ is $L^{\frac{n}{n-1}}$ differentiable a.e., see [1, Theorem 3.83]. Recently the notion functions of bounded variation has been extended to the class of metric measure spaces with doubling measure that admit a Poincaré inequality, see [2]. It would be interesting to see whether under this setting functions of bounded variation are $L^{q}$-differentiable for some values of $q$.

Remark 4.6. Let us observe that if the space $(X, d, \mu)$ does not support a Poincaré inequality the space $N^{1, p}$ could be quite nasty. For example, if $X$ is a totally disconnected subset of $\mathbb{R}^{n}$ then $N^{1, p}(X)$ simply coincides with $L^{p}$ and thus it could be that a $f \in N^{1, p}$ is nowhere differentiable in such cases.

If we want to obtain differentiability results for Sobolev functions on spaces which do not necessarily satisfy a Poincaré inequality it is useful to recall the definition of Sobolev space in the sense of Hajłasz as follows. 
Definition 4.7. Let $(X, d, \mu)$ be a metric measure space. For each $p>1$ we let $M^{1, p}(X)$ be the Hajłasz space which is the space of functions $f \in L^{p}(\mu)$ for which there exists $g \in L^{p}(\mu)$ so that for $\mu$-a.e. $x, y \in X$,

$$
|f(x)-f(y)| \leq d(x, y)(g(x)+g(y)) .
$$

Corollary 4.8. Let $\mu$ be a doubling measure. If $p>Q$ and $p \geq 1$ where $Q$ as in (2.4), then each function $f \in M^{1, p}$ has a locally Hölder continuous representative. Moreover, if $X$ admits a strong measurable differentiable structure, then that representative is differentiable $\mu$-a.e.

Proof. Let $f \in M^{1, p}$ and let $g \in L^{p}$ so that (4.8) of Definition 4.7 is satisfied. To show that $(f, g)$ satisfy a $(1, p)$-Poincaré inequality, fix a ball $\mathbf{B}$ in $X$. We than have for $\mu$-a.e. $x, y \in \mathbf{B}$ that

$$
|f(x)-f(y)| \leq d(x, y)(g(x)+g(y)) .
$$

If we integrate with respect to $x \in \mathbf{B}$ and then with respect to $y \in \mathbf{B}$ we obtain (see Theorem 3.1 in [15]):

$$
\begin{aligned}
f_{\mathbf{B}}\left|f-f_{\mathbf{B}}\right| d \mu & \leq 2 \cdot \operatorname{diam} \mathbf{B} f_{\mathbf{B}} g d \mu \\
& \leq 2 \cdot \operatorname{diam} \mathbf{B}\left(f_{\mathbf{B}} g^{p} d \mu\right)^{1 / p}
\end{aligned}
$$

which shows us that $(f, g)$ satisfy the Poincaré inequality. Applying Theorem 4.1 finishes the proof.

Corollary 4.9. Let $f:(X, d, \mu) \rightarrow(Y, \rho, v)$ be a quasi-conformal homeomorphism between metric measure spaces. If both $\mu$ and $\nu$ are $Q$-regular and if $X$ admits a strong measurable differentiable structure, then for each Lipschitz function $\phi: Y \rightarrow \mathbb{R}$ the function $\phi \circ f$ is differentiable $\mu$-a.e. in $X$.

Proof. Let us recall that a measure $\mu$ is $Q$-regular if there exists a constant $C>0$ such that

$$
\frac{1}{C} r^{Q} \leq \mu(\mathbf{B}(x, r)) \leq C r^{Q},
$$

for all $x \in X$ and $0<r<\operatorname{diam}(X)$.

Recall also from [14] the metric definition of quasiconformality of a homeomorphism $f: X \rightarrow Y$, according to which there exists a constant $K \geq 1$ such that for every $x \in X$

$$
\limsup _{r \rightarrow 0} \frac{\sup _{d(x, y) \leq r} \rho(f(x), f(y))}{\inf _{d(x, y) \geq r} \rho(f(x), f(y))} \leq K
$$

By Theorem 3.1, we need only check that $\operatorname{Lip}(\phi \circ f)$ is finite $\mu$-a.e. Indeed, for each $x \in X$ we have by (4.9) and (4.10)

$$
\begin{aligned}
\operatorname{Lip}(\phi \circ f)(x) & \leq \operatorname{LIP}(\phi) \cdot \limsup _{r \rightarrow 0} \frac{\sup _{d(x, y) \leq r} \rho(f(x), f(y))}{r} \\
& \leq C^{2} K \operatorname{LIP}(\phi) \cdot\left(\limsup _{r \rightarrow 0} \frac{\nu(f(\mathbf{B}(x, r)))}{\mu(\mathbf{B}(x, r)))}\right)^{\frac{1}{Q}} .
\end{aligned}
$$


Since for general homeomorphisms and $\mu$ doubling one has that the volume derivative

$$
\frac{d f_{\sharp} \nu}{d \mu}(x)=\lim _{r \rightarrow 0} \frac{v(f(\mathbf{B}(x, r)))}{\mu(\mathbf{B}(x, r))}
$$

exists as a finite number for $\mu$-a.e. $x$ we conclude that $\operatorname{Lip}(\phi \circ f)(x)<\infty$ for $\mu$-a.e. $x$ finishing the proof.

Remark 4.10. The assumption that both $X$ and $Y$ are $Q$-regular cannot be dropped. Indeed, let $\phi: \mathbb{R}^{n} \rightarrow \mathbb{R}$ be $\frac{1}{2}$-Hölder continuous function which is nowhere differentiable. Let $X$ be $\mathbb{R}^{n}$ with the Euclidean metric $d_{\mathbb{E}}$ and $Y$ be $\mathbb{R}^{n}$ with the metric $d_{\mathbb{E}}^{1 / 2}$. In this situation, $X$ is $n$-regular and $Y$ is $2 n$-regular. The map $f: X \rightarrow Y$ as $f(x)=x$ is quasi-symmetric and $\phi: Y \rightarrow \mathbb{R}$ is Lipschitz on $Y$, but $\phi \circ f$ is nowhere differentiable on $X$.

\section{References}

[1] Ambrosio, L., Fusco, N., and Pallara, D. Functions of Bounded Variation and Free Discontinuity Problems, Clarendon Press, (2000).

[2] Ambrosio, L., Miranda, M., and Pallara, D. Special functions of bounded variation in doubling metric measure spaces, preprint.

[3] Balogh, Z.M. and Csörnyei, M. Scaled oscillation and regularity, preprint, (2004).

[4] Björn, J. Poincaré inequalities for powers and products of admissible weights, Ann. Acad. Sci. Fenn. Math., 26(1), 175-188, (2001).

[5] Cheeger, J. Differentiability of Lipschitz functions on metric measure spaces, Geom. Funct. Anal., 9, 428-517, (1999).

[6] DeVore, D.A. and Sharpley, R.C. Maximal functions measuring smoothness, Mem. Am. Math. Soc., 47(293), 1-115, (1984).

[7] Evans, L.C. and Gariepy, R.F. Measure Theory and Fine Properties of Functions, CRC Press, Boca Raton, (1992).

[8] Federer, H. Geometric Measure Theorie, Die Grundlehren der mathematischen Wissenschaften in Einzeldarstellungen, no. 153, Springer-Verlag, Berlin, Heidelberg, (1969).

[9] Franchi, B., Hajłasz, P., and Koskela, P. Definitions of Sobolev classes on metric spaces, Ann. Inst. Fourier, (Grenoble), 49(6), 1903-1924, (1999).

[10] Gol'dshtein, V, and Troyanov, M. Axiomatic theory of Sobolev spaces, Expos. Math, 19(4), 289-336, (2001)

[11] Hajłasz, P. Sobolev spaces in metric-measure spaces, to appear.

[12] Hajłasz, P. Sobolev spaces on an arbitrary metric space, Potential Anal., 5, 403-415, (1996).

[13] Heinonen, J. Lectures on Analysis on Metric Spaces, Springer-Verlag, New York, (2001)

[14] Heinonen, J. and Koskela, P. Quasiconformal maps in metric spaces with controlled geometry, Acta Math., 181, 1-61, (1998).

[15] Hajłasz, P. and Koskela, P. Sobolev met Poincaré, Mem. Am. Math. Soc., 145(688), (2000).

[16] Keith, S. A differential structure for metric measure spaces, to appear.

[17] Keith, S. Measurable differentiable structures and the Poincaré inequality, to appear.

[18] Keith, S. A differentiable structure for metric measure spaces, $\mathrm{PhD}$ dissertation, University of Michigan at Ann Arbor, Department of Mathematics, (2002).

[19] Koskela, P. and MacManus, P. Quasiconformal mappings and Sobolev spaces, Studia Math., 131, 1-17, (1998).

[20] Maly, J. A simple proof of the Stepanov theorem on differentiability almost everywhere, Exposition. Math., 17, 59-61, (1999).

[21] Shanmugalingam, N. Newtonian spaces: An extension of Sobolev spaces to metric measure spaces, Rev. Mat. Iberoamericana, 16(2), 234-279, (2000).

[22] Stein, E. Singular Integrals and Differentiability Properties of Functions, Princeton University Press, Princeton, (1970).

[23] Väisälä, J. Lectures on n-dimensional quasiconformal mappings, Lecture Notes in Mathematics, 229, SpringerVerlag, Berlin, (1971). 


\title{
Received April 13, 2004
}

Department of Mathematics, University of Bern, Sidlerstrasse 5, 3012 Bern Switzerland e-mail: zoltan.balogh@math-stat.unibe.ch

Department of Mathematics, University of Bern, Sidlerstrasse 5, 3012 Bern Switzerland e-mail: kevin.rogovin@math-stat.unibe.ch

Department of Mathematics, University of Bern, Sidlerstrasse 5, 3012 Bern Switzerland e-mail: thomas.zuercher@math-stat.unibe.ch

\author{
Communicated by Steven Krantz
}

